$11 \mid 1989$

Varia

\title{
La reconstitution de l'expérience du vide dans le vide
}

Introduction

Dominique Descotes

\section{OpenEdition}

\section{Journals}

Édition électronique

URL : http://journals.openedition.org/ccibp/467

DOI : 10.4000/ccibp.467

ISSN : 2493-7460

Éditeur

Centre international Blaise Pascal

Édition imprimée

Date de publication : 20 décembre 1989

Pagination : 2-3

ISSN : 0249-6674

\section{Référence électronique}

Dominique Descotes, «La reconstitution de l'expérience du vide dans le vide », Courrier du Centre international Blaise Pascal [En ligne], 11 | 1989, mis en ligne le 10 décembre 2015, consulté le 20 avril 2019. URL : http://journals.openedition.org/ccibp/467 ; DOI : 10.4000/ccibp.467

Ce document a été généré automatiquement le 20 avril 2019

Centre international Blaise Pascal 


\section{La reconstitution de l'expérience du vide dans le vide}

Introduction

Dominique Descotes

1 L'expérience du vide dans le vide est directement liée à celle du Puy-de-Dôme : partant de l'hypothèse que la suspension du mercure dans le tube barométrique est un effet de l'équilibre entre la colonne de mercure visible et la colonne d'air invisible, Pascal décide de la vérifier en faisant varier la pression atmosphérique par une élévation en altitude, afin d'observer si le mercure varie avec elle; mais d'après la lettre à Périer du 15 novembre 1647, il a déjà fait l'expérience d'un cas particulier, celui où, le vide ayant été réalisé autour du tube barométrique, la pression de l'air se trouve annulée et la colonne de vif-argent «tombe entièrement, sans qu'il lui reste aucune hauteur ni suspension ». C'est l'expérience du vide dans le vide.

2 Cette expérience, dont le P. Noël fournit un compte-rendu plus précis dans sa Gravitas comparata de 1648 n'est pas la seule connue : Pascal en a donné une seconde version dans le chapitre VI du Traité de la Pesanteur de la Masse de l'Air, et d'autres savants en ont fait autant, à des dates différentes.

3 Le problème de la réalisation effective de ces expériences touchant le vide a été soulevé en 1954 par Alexandre Koyré, dans sa conférence sur Pascal savant au colloque de Royaumont. Il faisait alors remarquer que Pascal n'avait pas toujours décrit ses expériences telles qu'il les avait réalisées, que, dans certains cas, il relatait sans doute des «expériences de pensée », c'est-à-dire purement imaginaires. Or l'expérience du vide dans le vide est de celles qui, dans cette perspective, demandent un examen attentif, à cause de sa difficulté.

4 On aperçoit donc l'intérêt des recherches que Kimiyo Koyanagi a menées, avec Akira Kurobé et Masao Uchida pour reconstituer, non pas dans l'abstrait, mais tout à fait concrètement, les expériences faites au XVII ${ }^{\mathrm{e}}$ siècle par Pascal et ses contemporains. Ces travaux se sont déroulés en deux phases : la première en 1978, au Laboratoire National de Métrologie du Japon, a abouti à la reconstitution du dispositif décrit par Pascal dans $L a$ Pesanteur de la Masse de l'Air par K. Koyanagi et A. Kurobé; la seconde, conduite avec 
Masao Uchida, a permis la réalisation de quatre dispositifs différents, celui de Pascal dans le Récit de la Grande Expérience, et ceux d'Auzoult, de Roberval et de Rohault; un compte rendu oral de ces travaux a été présenté à l'occasion de la vingt-neuvième assemblée de la Société Japonaise d'Histoire des Sciences en 1982.

Le principe de ces travaux consiste à tenter de reconstituer les expériences en suivant d'aussi près que possible les indications des textes, pour en mesurer les difficultés concrètes et chercher par quels procédés, dissimulés par la «stylisation " des comptes rendus, Pascal et ses amis ont pu en triompher. Pour reprendre une formule de Philippe Sellier, il s'agit de retrouver « Pascal au travail ». Ce sont les clichés pris au cours de ces reconstitutions qu'avec l'autorisation de leurs auteurs, nous publions ici. Ils témoignent, dans leur ensemble comme dans leur progression, d'un esprit de méthode qui ne manquera pas de frapper nos lecteurs.

6 La réussite même de ces reconstitutions montre d'ailleurs qu'en la matière la recherche n'est pas close : peut-être saurons-nous un jour si, comme le suppose K. Koyanagi, les contemporains de Pascal connaissaient un moyen d'obturer leurs tuyaux par des peaux assez solides pour résister à la pression du mercure, et si Pascal avait trouvé quelque procédé pour tourner cette difficulté. Il en résulte que, pour leurs auteurs, le succès de ces restitutions n'entraîne pas automatiquement qu'elles aient été effectivement réalisées à l'époque de Pascal, puisque K. Koyanagi pense que l'expérience relatée dans La Pesanteur de la Masse de l'Air est une expérience de pensée, et non une réalisation effective.

Dans les pages qui suivent, les clichés sont accompagnés du texte original de chaque relation, avec ses références dans l'édition des Euvres complètes de Pascal par Jean Mesnard, à l'exception du Traité de Physique de Rohault, dont le passage ad hoc est reproduit intégralement. Les figures sont dues aux auteurs des expériences, à l'exception des dessins de Duhem et de Thirion qui accompagnent les textes de la Gravitas comparata.

Pour suivre la chronologie des expériences, nous aurions dû adopter l'ordre suivant: d'abord l'expérience de Pascal rapportée par le P. Noël, qui est la plus ancienne ; puis les expériences d'Auzoult et de Roberval ; celle de La Pesanteur de la Masse de l'Air, et celle de Rohault en dernier lieu. Mais cette présentation conduirait à démembrer l'ensemble de quatre expériences réalisées par M. Uchida, et à briser la continuité des travaux de restitution. Il fallait donc commencer par le second dispositif de Pascal, qui n'est pas le plus ancien du point de vue chronologique. Le lecteur n'aura aucune peine à rétablir la succession historique.

\section{INDEX}

Mots-clés : vide

Keywords : vacuum 
AUTEUR

DOMINIQUE DESCOTES

CERHAC. Université Blaise Pascal 